\title{
Mesoflowers: A New Class of Highly Efficient Surface-Enhanced Raman Active and Infrared-Absorbing Materials
}

\author{
Panikkanvalappil Ravindranathan Sajanlal and Thalappil Pradeep ( $\varangle)$ \\ DST Unit on Nanoscience (DST UNS), Department of Chemistry and Sophisticated Analytical Instrument Facility, Indian Institute of \\ Technology Madras, Chennai 600 036, India \\ Received: 22 December 2008/Revised: 5 February 2009/Accepted: 5 February 2009 \\ (C) Tsinghua University Press and Springer-Verlag 2009. This article is published with open access at Springerlink.com
}

\begin{abstract}
A method for the synthesis of a new class of anisotropic mesostructured gold material, which we call "mesoflowers", is demonstrated. The mesoflowers, unsymmetrical at the single particle level, resemble several natural objects and are made up of a large number of stems with unusual pentagonal symmetry. The mesostructured material has a high degree of structural purity with star-shaped, nano-structured stems. The mesoflowers were obtained in high yield, without any contaminating structures and their size could be tuned from nano- to meso-dimensions. The dependence of various properties of the mesoflowers on their conditions of formation was studied. The near-infrared-infrared (NIR-IR) absorption exhibited by the mesoflowers has been used for the development of infrared filters. Using a prototypical device, we demonstrated the utility of the gold mesoflowers in reducing the temperature rise in an enclosure exposed to daylight in peak summer. These structures showed a high degree of surface-enhanced Raman scattering (SERS) activity compared to spherical analogues. SERS-based imaging of a single mesoflower is demonstrated. The high SERS activity and NIR-IR absorption property open up a number of exciting applications in diverse areas.
\end{abstract}

\section{KEYWORDS}

Mesoflowers, gold nanoparticles, seed-mediated growth, IR absorption, surface-enhanced Raman scattering (SERS)

\section{Introduction}

Anisotropic metal meso[1, 2]/nanostructured materials with complex shapes are fascinating, since materials in this size regime are expected to exhibit numerous extraordinary properties which are not possible for spherical analogues. The chemical and physical properties of mesostructured materials depend on their geometry and hierarchical organization. The success of nanotechnology depends not only on the development of new nanoscale materials but also their integration over meso- (10 nm to micrometers) and microscale to combine properties which are desirable for the development of future nanodevices.

Noble metal particles at the nanometer to micrometer range have been used in heterogeneous catalysis and electrocatalysis. Complexity in morphology adds more attractive optical and spectroscopic properties. Recent examples of such

Address correspondence to pradeep@iitm.ac.in 
complex materials include nanorods [3-5], prisms or triangles [6-9], wires [10,11], multipodes [12], stars [13], tadpoles [14], and variety of other structures. Crystals of spherical nanoparticles, the so called superlattices $[15,16]$ corresponding to two- and three- dimensional arrangement of nanoparticles, are often anisotropic and show interesting properties. Anisotropic optical absorption [17-20] and chemical reactivity [21] are some of the properties of gold nanorods currently under investigation. Intense surface plasmon resonance (SPR) and associated photophysical properties have been utilized in waveguides [22]. Amplification of electromagnetic fields at the vertices [23] of these meso/nanostructured materials may be utilized for single molecule detection in both Raman and fluorescence spectroscopy. The unusual near-infared (NIR) absorption of noble metal anisotropic particles has made them promising candidates for cancer therapy [24]. The diverse possibilities mentioned above make an exploration of novel anisotropic mesostructured materials relevant. Developing a reproducible and viable synthesis of such structures, devoid of other contaminating structures-principally spherical particles_-is indeed a difficult task.

Here we report a new class of one such structure of gold, which we name "mesoflowers", which are highly irregular, requiring several structural parameters to describe them accurately. However, our synthetic procedure yields mesoflowers of highly uniform shape. The synthetic process has been optimized to yield gold mesoflowers of varying sizes ranging from nano- to meso-dimensions, while preserving the structure. Synthesis of highly uniform nano/mesoflowers with a high degree of structural purity without even a few contaminating structures, and thus requiring no purification, makes this synthetic approach unique. A mesoflowercoated glass substrate showed very good surfaceenhanced Raman scattering (SERS) activity with an enhancement factor of the order of $10^{\circ}$. Such a substrate also showed optical absorption in the visible-NIR window suggesting applications in infrared filters. We demonstrated the viability of the mesoflowers as optical filters by a simple real-time experiment. A preliminary study showed that the mesoflower-coated glass substrates are capable of absorbing the NIR-IR fraction of the sunlight, which reduces the temperature rise of the enclosure. Thus, this material can be used as a heat-absorbing optical coating for windows. It is attractive that a simple synthetic method such as the one presented here can produce such beautiful and technologically relevant meso / nanoscale objects in high yield.

\section{Results and discussion}

In a typical synthesis of gold mesoflowers (details are given in the Experimental section), Au/oligoaniline nanoparticles synthesized as per our earlier report [25] were added to a growth solution containing a mixture of cetyltrimethylammonium bromide $(\mathrm{CTAB}), \mathrm{Au}^{3+}, \mathrm{AgNO}_{3}$, and ascorbic acid. The unstirred solution was maintained at $80{ }^{\circ} \mathrm{C}$ for $1 \mathrm{~h}$, resulting in the formation of gold mesoflowers. It is important to keep the solution at this temperature. The resultant solution was centrifuged at $4000 \mathrm{rpm}$ for $5 \mathrm{~min}$ and the sand-colored gold mesoflower residue was collected. From the large area field emission scanning electron microscope (FESEM) image of a monolayer of gold mesoflowers anchored on an indium tin oxide (ITO) glass plate, one can see that the synthesis yields regular structures (Fig. 1(a)). In fact no spherical or other structures were found. All the mesoflowers showed the same morphology. The FESEM image (Fig. 1(b)) of a single mesoflower reveals the highly complex anisotropic nature of the mesoflowers which are biomimetic structures, resembling aloe vera or pineapple. Each mesoflower is made up of a large number of spiky stems, which are projecting outward from the core, in all directions. From the FESEM images of the mesoflowers, it was found that the number of stems on each mesoflower varies from particle to particle. These stems make the mesoflowers three dimensional. We investigated several individual mesoflowers and all the particles observed were found to have more than 10 stems, and ranging up to 20. A typical synthesis (using $20 \mathrm{~mL}$ growth solution, $3.3 \mathrm{mg} \mathrm{Au}{ }^{3+}$ ) makes $2.9 \mathrm{mg}$ of the material and a photograph of the mesoflower powder in the solid state is shown in the inset of Fig. 1(b).

In order to study the spatial distribution of gold 
in the mesoflower, elemental mapping of a single mesoflower was carried out using energy dispersive analysis of X-rays (EDAX). Figure 2(a) shows an $\mathrm{Au} \mathrm{M} \alpha$-based image. Elemental maps of $\mathrm{Au} L \alpha, \mathrm{N}$ $\mathrm{K} \alpha$ and Si K $\alpha$ were also measured (Fig. S-1 in the Electronic Supplementary Material (ESM)). From the EDAX analysis, it was confirmed that the mesoflower is almost completely made of gold. Figure 2(b) shows a magnified SEM image of a single stem of the mesoflower. Each stem has ridges along its corners which give rise to a unique morphology. The presence of five edges gives a star-shaped appearance to the stem when it is viewed from the top and ridges along the corners of the stems give a stacked appearance. A view of the stem from its top appears as a star of edge length $\sim 400$ nm (Fig. 2(c)). It is likely that the growth starts from the 3 $\mathrm{nm}$ particles seen at the apex of the growing stem. A model of one such star-shaped stem is shown in Fig. 2(d). Top views of a stem at different rotation angles (Fig. S-2 in the ESM) show the symmetrical nature of this star. These stems show an unusual pentagonal symmetry and maintain an angle of $\sim 72^{\circ}$ between each successive lobe of the stars. A typical large area SEM image of the mesoflowers clearly illustrates that the stems possess simple and remarkably well-defined uniform starlike structures with five vertices (Fig. S-3 in the ESM). The stems bear a strong resemblance to a hierarchical array of scales or plate-like subunits which themselves powder have the shape of stars, forming a pyramid of stars (Fig. S-3 in the ESM). Even though the mesoflower particle as a whole does not possess any symmetry, we see a complex five-fold symmetry along the major axis of the stem of the mesoflowers. All the structural features of these flowers are in the nano/meso regime and therefore we suggest the name "mesoflowers". Although various structures of flowers and stars have

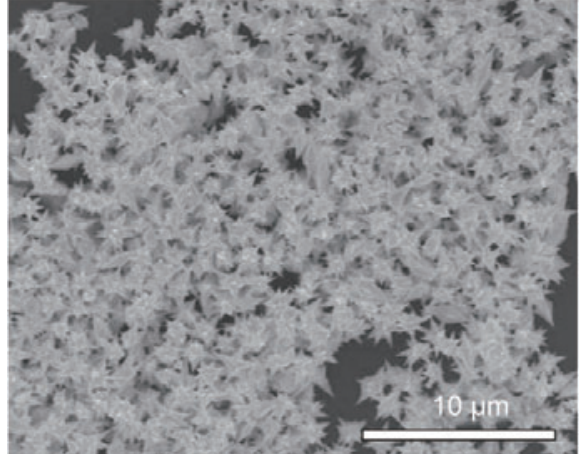

(a)

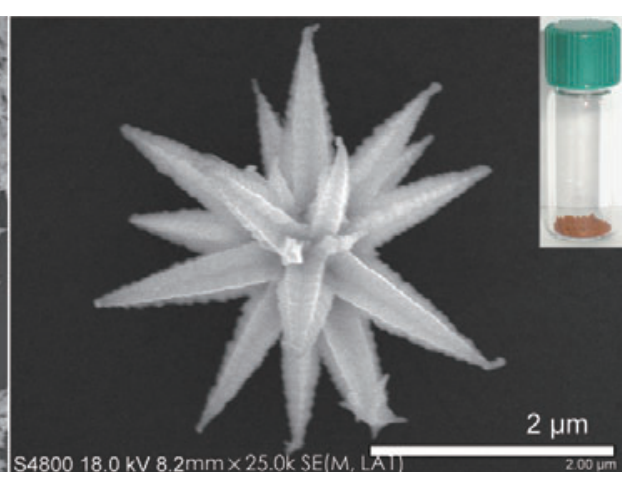

(b)
Figure 1 (a) Large area FESEM image of gold mesoflowers anchored on a conducting glass substrate; (b) FESEM image of a gold mesoflower. The inset shows a photograph of the mesoflower

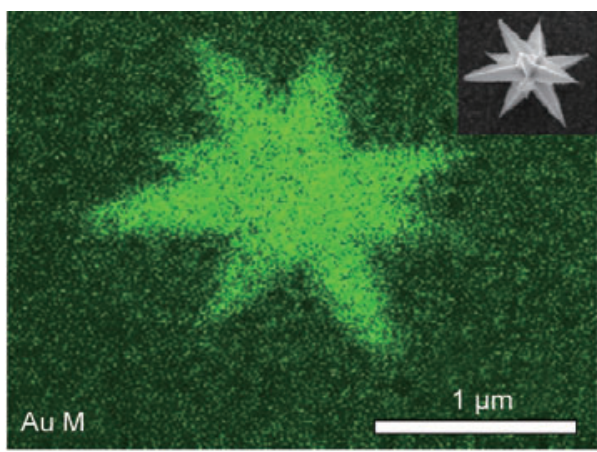

(a)

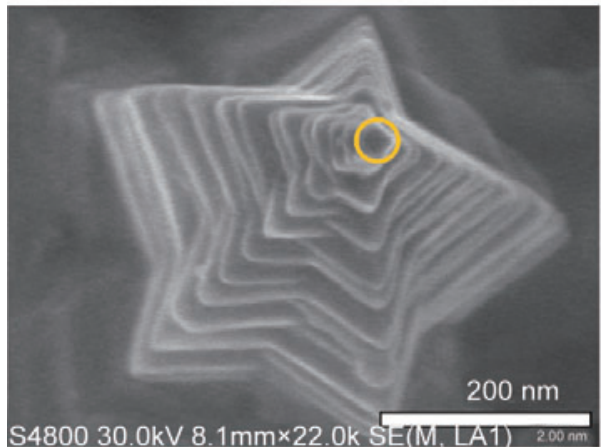

(c)

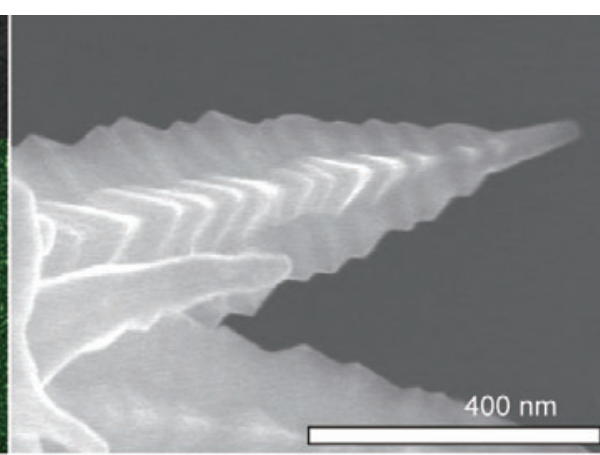

(b)

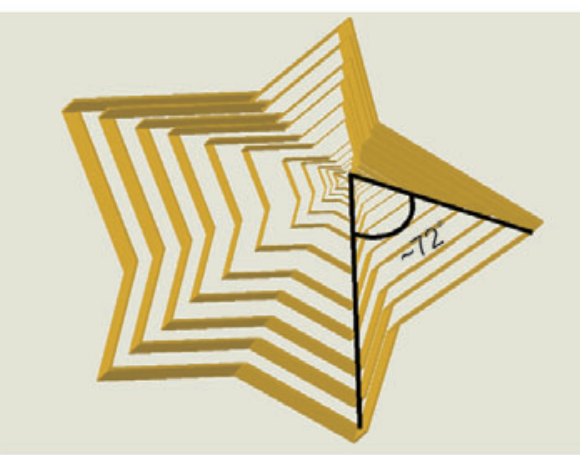

(d)
Figure 2 (a) An EDAX image of $A u$ M $\alpha$ taken from the mesoflower shown in the inset; (b) an enlarged FESEM image of a single stem of the mesoflower showing ridges along the edges; (c) top view of a single stem of the mesoflower showing the pentagonal structure and a nanoparticle on the apex (marked) and (d) a corresponding model 
been previously reported [26-32], none of them have the same structure and unusual uniformity of these mesoflowers. The spiky stems and the abundance of such features in each mesoflower, the pentagonal symmetry of the plate-like units and the simplicity of the synthetic approach giving uniformly-shaped structures in large yield, make these materials unique. It is possible to synthesize mesoflowers on a gram scale without any contaminating structures, and the material can be preserved in the powder form. Even though there are some structural differences between the mesoflowers, such as the number and the length of the stems, the number of plate-like subunits, etc., the main attraction of the mesoflowers is their structural purity. We note that there are numerous structural parameters for the mesoflowers and they are not absolutely identical. The stems in each mesoflower show the unusual pentagonal symmetry, with both shorter and longer stems present inside the same flower. It is also possible to see larger mesoflowers through an optical microscope (Fig. S-4 in the ESM).

Furthermore, the size of the assynthesized three-dimensional mesoflowers can be controlled by altering the amount of the precursor $\mathrm{Au}$ / oligoaniline nanoparticles added. Mesoflowers larger than $1 \mu \mathrm{m}$ in size were formed when $2 \mathrm{~mL}$ of the precursor nanoparticles were added to $20 \mathrm{~mL}$ of the growth solution under the optimized experimental conditions. Figure 3(a) shows the transmission electron microscopy (TEM) image of such a single gold mesoflower. The average size of the mesoflowers was 1-2 $\mu \mathrm{m}$. The size of the mesoflowers was found to decrease when the amount of seed solution added was increased to 5 $\mathrm{mL}$, giving mesoflowers of length $0.5-1$ $\mu \mathrm{m}$ (Fig. 3(b)). The size further decreased to $\sim 150 \mathrm{~nm}$ by the addition of $6 \mathrm{~mL}$ of seed solution into the growth solution (Fig. 3(c)). This reveals the flexibility of our synthetic approach in tuning the size of the meso/ nanoflowers. At higher seed concentrations, a large number of seed particles will take part in the growth process and the amount of gold ions in the growth solution will not be sufficient as they will consumed before the complete growth of individual particles. A lattice-resolved TEM image of a stem of a mesoflower is shown in Fig. 3(d) and the corresponding selected area electron diffraction (SAED) is shown in the inset. The gold mesoflowers exhibit a lattice spacing of $2.35 \AA$, which corresponds to the (111) plane of gold. Large area TEM and SEM images of the mesoflowers of different sizes are shown in Fig. S-5 (in the ESM). The lengths of mesoflowers of different sizes were measured from the line profile taken along the TEM images of the mesoflowers (Fig. S-6 in the ESM). The XRD patterns of the mesoflowers (Fig. S-7 in the ESM) showed the characteristic (111), (200), (220), (311), and (222) reflections of face-centered cubic gold.

It is known that the temperature plays an important role in determining the morphology of nanoparticles [33-36] and the effect of varying

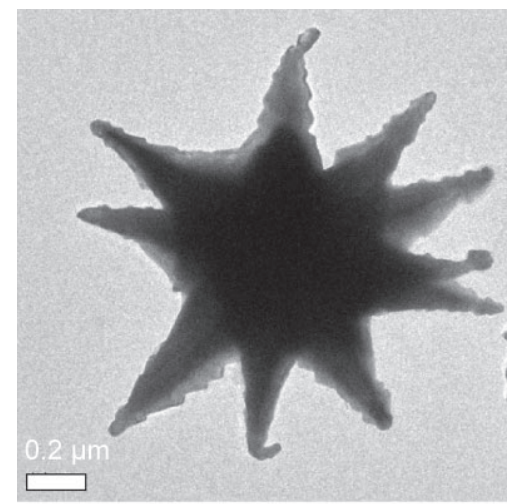

(a)

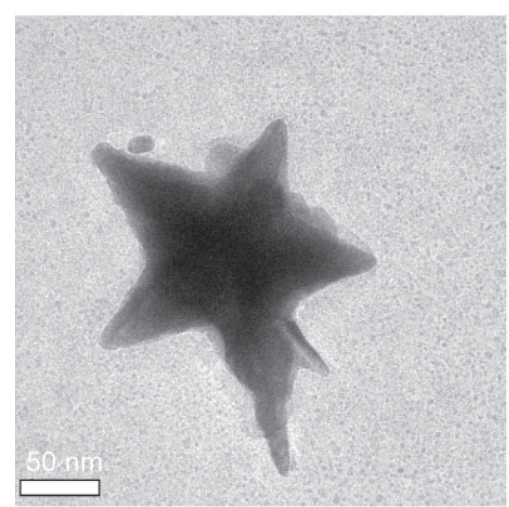

(c)

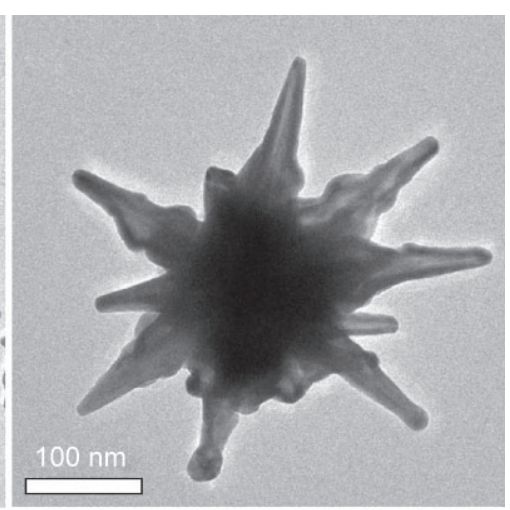

(b)

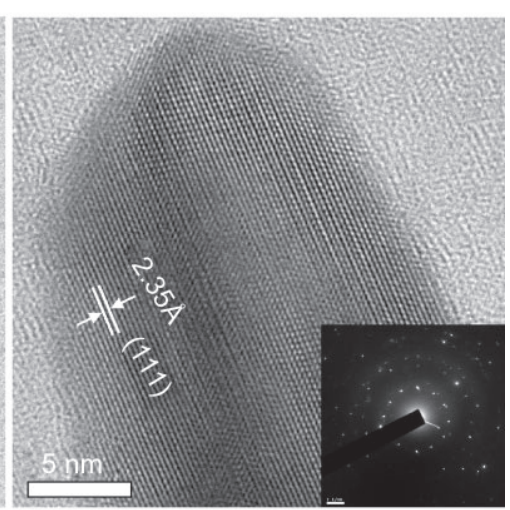

(d)
Figure 3 TEM images of the mesoflowers of different sizes formed when varying amounts of seed solutions were added, such as (a) $2 \mathrm{~mL}$, (b) $5 \mathrm{~mL}$, and (c) $6 \mathrm{~mL}$, into $20 \mathrm{~mL}$ of the growth solution; (d) a lattice-resolved TEM image taken from the tip of a mesoflower shown in (a). An SAED pattern taken from the tip of the mesoflower is shown in the inset of (d) 
temperature on the formation of the mesoflowers was therefore investigated. When the reaction was carried out at $0{ }^{\circ} \mathrm{C}$, the morphology changed drastically to a meatball-like mesostructure. Compared to the mesoflowers formed under the same concentration at high temperature, the length of the spiky stems formed in the meatball-like mesostructures was short. The TEM image of a single meatball-like mesostructure and the corresponding lattice-resolved image are presented in Fig. S-8 (in the ESM). Poorly grown small spikes, but no star-shaped stems can be seen. An SEM image (Fig. S-9 in the ESM) also revealed the same features. At low temperature, instead of developing stems, the core of the nanoparticles grows rapidly. This material showed an intense, but featureless absorption in the NIR region (Fig. S-9 in the ESM). From EDAX and XRD (Fig. S-9 in the ESM), it was confirmed that the material is also essentially composed of pure gold.

The formation of mesoflowers largely depends on the morphology of the precursor $\mathrm{Au} /$ oligoaniline nanoparticles. The precursor nanoparticles are raspberry-like aggregates of smaller nanoparticles which are embedded in the oligoaniline matrix [25]. Even though all the nanoparticles in the spherical aggregate can act as nucleation sites for further growth, only those which have exposed surfaces, where the oligoaniline is attached loosely, will undergo further growth. The number of such particles with exposed surfaces is the key factor which determines the number of stems in the mesoflower. Here the temperature plays an important role in creating the nucleation sites. Heating during growth may cause the rupturing of the oligoaniline coating on the nanoparticles to some extent and this may create more exposed nucleation sites. The formation of the meatball-like structure with poorly grown thorns at ice-cold temperature clearly demonstrates the effect of varying temperature. Figure 4(a) shows a TEM image of Au/oligoaniline seed nanoparticles. From the lattice-resolved image, it is clear that the smaller particles present inside the
Au/oligoaniline seed are multi-twinned. The gold (111) plane with $d$ spacing of $2.35 \AA$ is marked in the lattice-resolved image (Fig. 4(b)). The presence of multiple twinning in these seed particles may provide favorable sites for further growth leading to the anisotropic stems $[13,37]$. The $\mathrm{Ag}^{+}$ions adsorbed on certain crystallographic planes of the growing seed nanoparticles may not allow the isotropic growth of these seed nanoparticles into large spheres [3]. The formation of mesoflowers was not observed in the absence of $\mathrm{Ag}^{+}$in the growth solution. This is

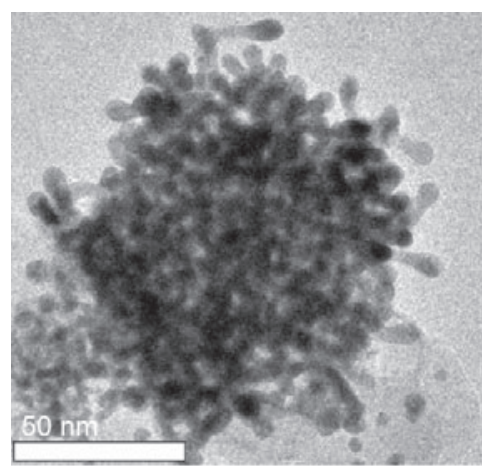

(a)

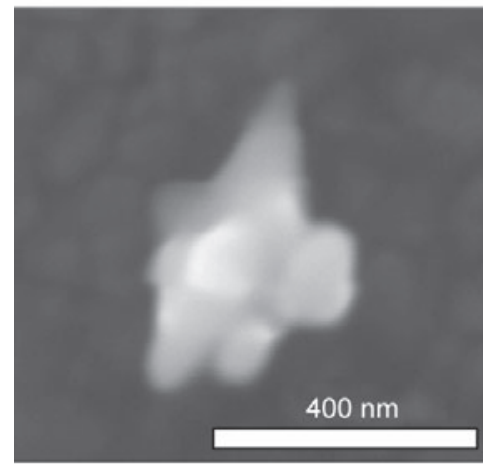

(c)

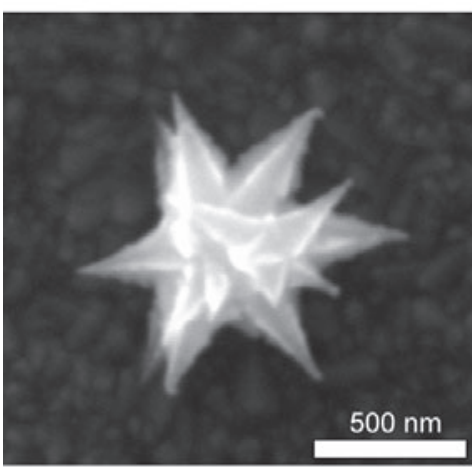

(e)

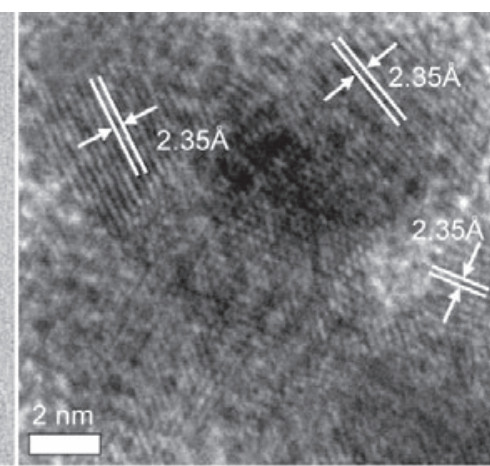

(b)

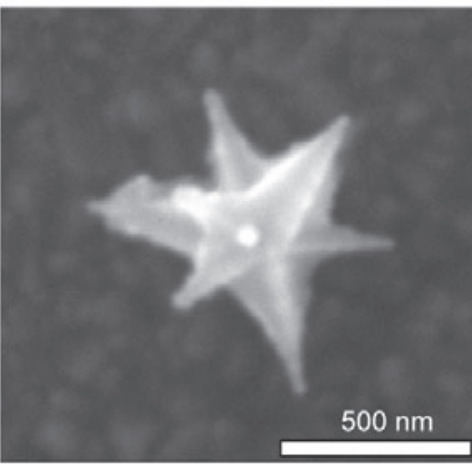

(d)

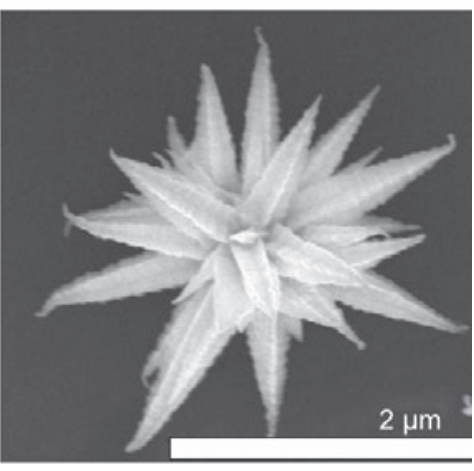

(f)
Figure 4 (a) TEM image of the Au/oligoaniline seed nanoparticle and (b) the lattice-resolved image taken from the seed particle. (c), (d), (e) and (f) are SEM images of the mesoflowers formed at various stages of growth $-2,5,10$, and 60 min, respectively 
similar to previous reports of nanorod growth [3]. Although the mechanism of formation of the starlike pyramids is not completely clear at this stage, the branches are most likely the result of growth due to the multiple twinning in the seeds.

In order to trace the reaction pathway, we analyzed the particles formed at various stages of the reaction. Figures 4(c)-(f) show SEM images of the intermediate structures formed at different stages of the reaction. To collect the intermediate particles, the reaction was stopped after 2, 5, 10, and $60 \mathrm{~min}$ and the resultant solutions were centrifuged at 10,000 rpm in order to remove excess CTAB and other ions. The precipitate was washed with distilled water and analyzed using SEM. Within $5 \mathrm{~min}$ of the reaction, the seed particles formed the flower-like morphology in all its microscopic details, but they were smaller in size. The stems maintained their unusual pentagonal morphology. This indicates that the growth of the seed particles into the mesoflowers is very fast. Assembly of smaller structures such as nanoplates to form stems is unlikely on this time scale. We could isolate mesoflowers of size $<500 \mathrm{~nm}$ after reaction for $5 \mathrm{~min}$. This suggests that tuning the size of the mesoflowers as a function of time is possible. We believe that the star-shaped pyramids are formed by the selective and step-wise growth of the multitwinned seeds whose subsequent development leads to the formation of the five-edged stem, which gradually propagates resulting in a hierarchical pyramid of stars. The absence of the star-shaped platelets at any intermediate stages of the reaction ruled out the possibility of such independent platelets being formed first, with subsequent assembly leading to the stems. We did explore the grid (in TEM) and the sample stub (in SEM) in great detail at various stages of growth and could not locate any platelets. If platelets had been formed, their complete disappearance is unlikely, especially in the first few minutes of synthesis.

The elemental composition of the mesoflowers was studied using X-ray photoelectron spectroscopy (XPS). Figure 5 shows a wide scan XPS spectrum of the mesoflowers and the parent $\mathrm{Au}$ /oligoaniline seed particles. The XPS spectrum of the mesoflower (Fig. $5(\mathrm{a}))$ revealed that it is composed of gold along with the expected surface contaminants. The prominent peaks seen are $\mathrm{Au} \mathrm{4f}, \mathrm{Au} \mathrm{4d}, \mathrm{C} 1 \mathrm{~s}$ and $\mathrm{O} 1 \mathrm{~s}$. An enlarged XPS spectrum in the Au $4 \mathrm{f}$ region is shown in the inset of Fig. 5(a). The presence of $A u 4 f_{7 / 2}$ and $\mathrm{Au} 4 \mathrm{f}_{5 / 2}$ peaks, at $\sim 83.9$ and $\sim 87.8 \mathrm{eV}$, respectively, confirms the existence of gold in its metallic form. The intensities of $\mathrm{C} 1 \mathrm{~s}$ and $\mathrm{O} 1 \mathrm{~s}$ are weak and can be attributed to the thin surfactant cover. CTAB is unlikely to be present at the detection level of XPS as no $\mathrm{Br}$ feature was detected (however, it is seen in mass spectrometry). In the case of Au/oligoaniline seed particles, the Au features in the XPS spectrum were masked by the high oligoaniline content (or due to the surface sensitivity of the technique) and

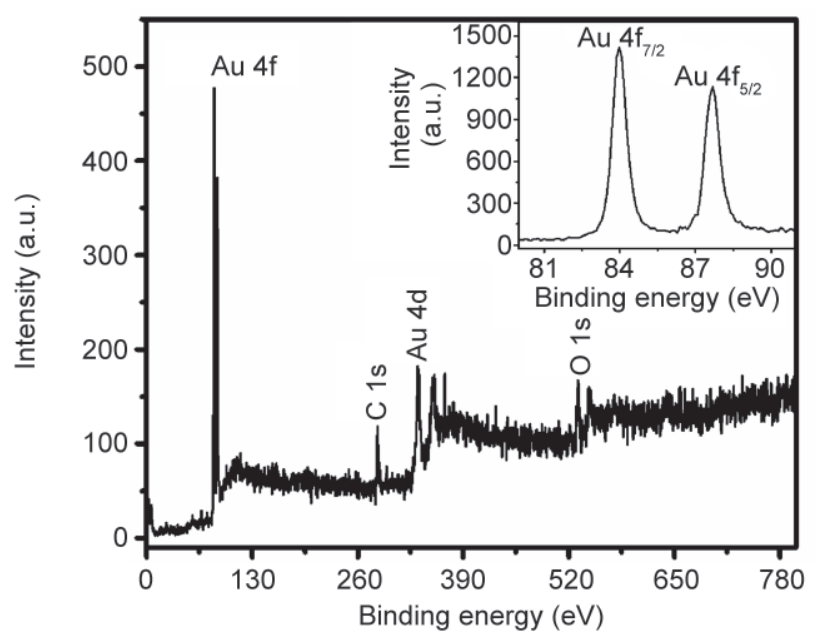

(a)

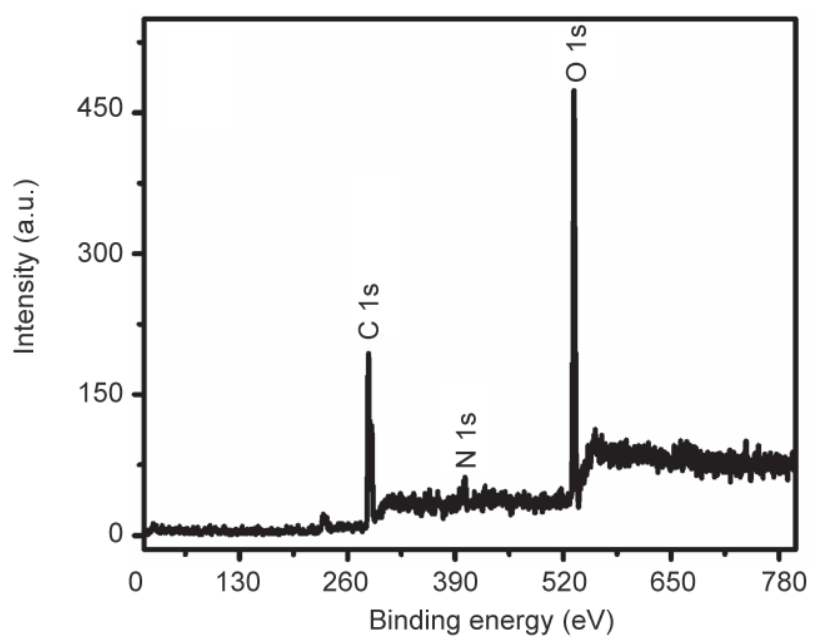

(b)

Figure 5 XPS spectrum collected from (a) the gold mesoflower of size 1-2 $\mu \mathrm{m}$ and (b) Au/oligoaniline seed particles. The inset in (a) shows the enlarged spectrum in the $\mathrm{Au} 4 \mathrm{f}$ region 
the peaks due to the polymer were prominent (Fig. $5(b))$. The presence of $C 1 \mathrm{~s}$ and $N$ 1s suggests the existence of oligoaniline in the seed particles. The $\mathrm{C}$ 1s peak of Au/oligoaniline is split into three peaks in the binding energy range between 284 and 289 $\mathrm{eV}$. The enlarged XPS spectrum of C 1 s of the seed particles is shown in Fig. S-10 (in the ESM). The main peak at $284.5 \mathrm{eV}$ is attributed to the $\mathrm{C} 1 \mathrm{~s}$ of the oligoaniline originating from $\mathrm{C}=\mathrm{C}$ and $\mathrm{C}-\mathrm{H}$ bonds of the aromatic ring. The other two peaks situated at higher binding energy (286.5 and $288.8 \mathrm{eV}$ ) may be due to the $\mathrm{C}$ atoms directly attached to the $\mathrm{N}$ atom of the oligoaniline polymeric chain [38,39]. Thus the observation of multiple peaks in the $\mathrm{C} 1 \mathrm{~s}$ region confirms the presence of oligoaniline in the seed particle, although it is absent in the mesoflower. The oligoaniline is expected to be protonated under the acidic conditions employed and that is reflected in the $\mathrm{N}$ 1s binding energy $(401.0 \mathrm{eV})$.

Laser desorption ionization mass spectra (LDI MS) of the Au/oligoaniline seed nanoparticles and mesoflowers are shown in Fig. 6. In the case of $\mathrm{Au}$ /oligoaniline seed particles, two series of peaks separated by $m / z 91$ were observed (Fig. 6(a)). This indicates the presence of oligoaniline. The peaks correspond to $-\left(\mathrm{C}_{6} \mathrm{H}_{4}-\mathrm{NH}\right)_{n}$, where $n$ corresponds to 1 to 8 . Corresponding peaks in the two series differ by $m / z 15$ (due to the loss of terminal amine, $-\mathrm{NH}$ ) [25]. In the case of the mesoflowers, although the $\mathrm{CTAB}$ concentration is low, it is the major peak $(\mathrm{m} / \mathrm{z}$ $285)$ in the positive ion spectrum, as is typical of any quarternary ammonium ion-containing material (Fig. 6(b)). This is due to the high sensitivity of LDI for preformed ions. The very weak peaks appearing at $m / z 365$ and 729 may be due to the presence of trace amounts of oligoaniline (tetramer and octamer, respectively) adsorbed on the mesoflower even after washing. This clearly indicates that the oligoaniline is almost totally absent on the surface of the mesoflower, consistent with the XPS results. The peaks at $m / z 197,394$, and 591 in the negative mode LDI MS (Fig. 6(c)) correspond to $\mathrm{Au}, \mathrm{Au}_{2}$, and $\mathrm{Au}_{3}$ ions, typical of laser desorption of metallic gold.

Because of the large anisotropy in the structure of the mesoflower, the material showed a featureless absorption spectrum in the NIR-IR region. Figure S-11

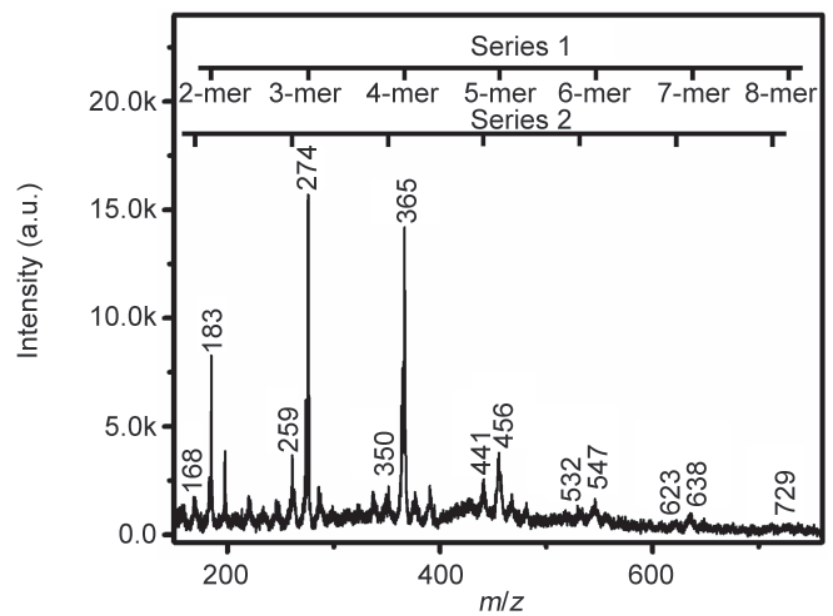

(a)

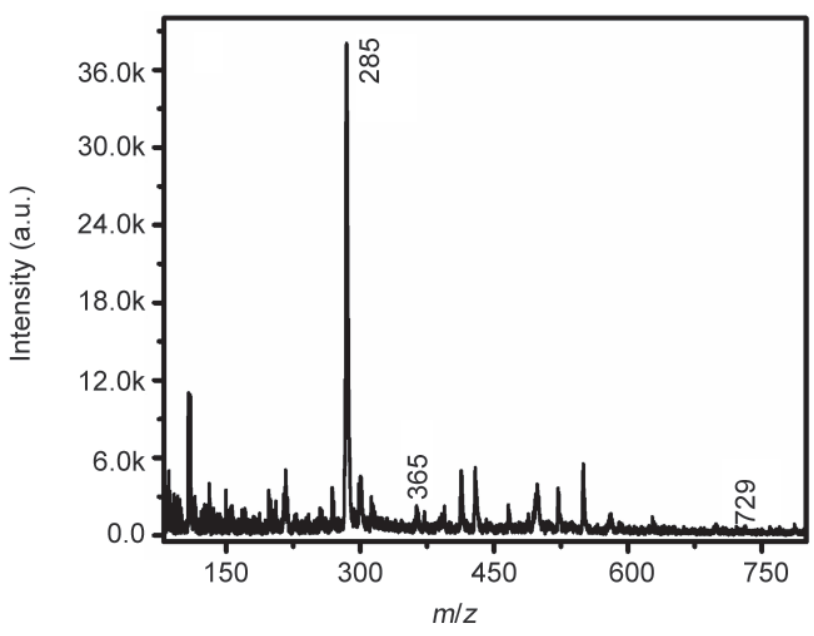

(b)

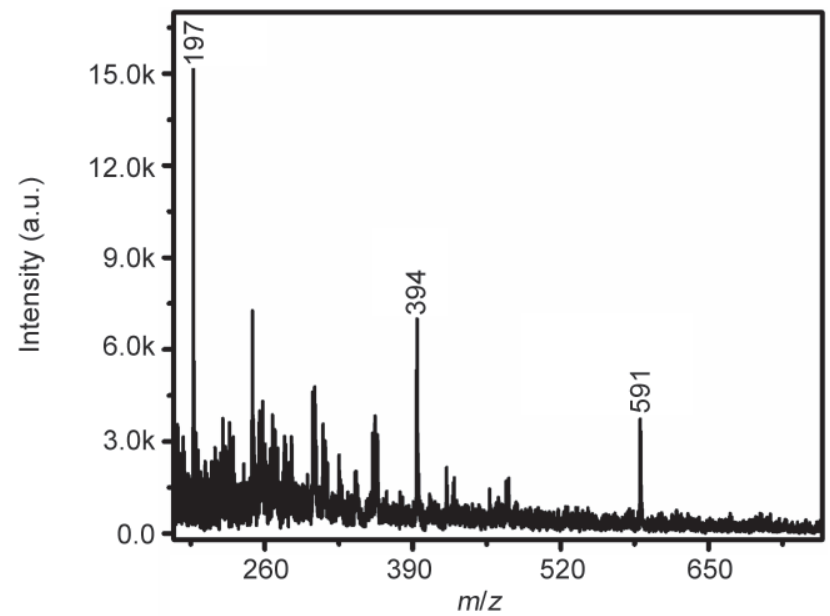

(c)

Figure 6 (a) LDI MS of the Au/oligoaniline seed taken in the positive mode. The two series of oligomeric peaks are indicated. Peaks in series 2 occur at $m / z 15$ lower than the corresponding peaks in series 1. (b) and (c) are the LDI MS of the mesoflower taken in positive and negative modes, respectively. The peak at $m / z 285$ in (b) due to the cetyltrimethylammonium ion is enhanced due to its high sensitivity 
(in the ESM) shows the optical absorption spectra of dispersions of mesoflowers of different sizes and the precursor $\mathrm{Au} /$ oligoaniline nanoparticles. Since water absorbs in the NIR region, it was difficult to observe the absorption characteristics of the mesoflower suspension beyond $1400 \mathrm{~nm}$. In order to overcome this, the mesoflowers were coated onto a glass substrate $(3 \mathrm{~cm} \times 3 \mathrm{~cm} \times 0.2 \mathrm{~cm})$ and the absorption spectrum was measured in the UV-vis-NIR region (in the same way as for the solution phase measurement, as described in the Experimental section). The mesoflower-coated glass substrate showed a noticeable red shift in its absorption maximum as the particle size increased. This was evident from the UVvis-NIR spectra of monolayer-coated glass plates (Fig. $7(\mathrm{a})$ ). Mesoflowers of size $\sim 0.5 \mu \mathrm{m}$ showed a broad absorption maximum around $1400 \mathrm{~nm}$ whereas the absorption of $\sim 1.5 \mu \mathrm{m}$ mesoflowers extended beyond $2500 \mathrm{~nm}$. It is known that the optical absorption and scattering of nanoparticles are strongly dependent on their size, shape, and composition. Processes such as absorption, scattering, and extinction cross section of particles of arbitrary shapes have been extensively studied using Mie theory and the discrete dipole approximation (DDA) [40-42]. Because of the large size of the mesoflowers, along with the absorption of electromagnetic radiation, scattering is also expected. As in the case of the nanorods and nanostars, absorption in the NIR-IR region is likely because of the longitudinal surface plasmon resonance of the elongated stems of the mesoflowers [13].

It has been recognized that NIR-absorbing films based on gold can be used as an alternative to reflective coatings for blocking IR radiation [43]. Gold-based approaches are highly efficient and can be more economically viable than other methods. The viability of mesoflowers as optical filters was checked by a real-time measurement. For the IR absorption study, we first made glass substrates (15 $\mathrm{cm} \times 15 \mathrm{~cm} \times 0.5 \mathrm{~cm})$ on which the mesoflowers were immobilized. We checked the optical transparency of these mesoflower-coated glass substrates. Figure 7(b) shows the transmittance spectra of the mesoflowercoated glass substrate that we used for the IR absorption study. Even after two layers of coating, the bilayer of mesoflower-coated glass substrate showed around $80 \%$ transmittance in the visible region (Fig. $7(b))$. Such high transparency in the visible region together with significant NIR absorption makes them promising candidates [43] for developing IR absorbing materials and thin films.

Figure 7(c) shows a photograph of the appparatus used for the real-time measurements. Our experimental set-up consists of square cardboard boxes with exposed top and bottom sides. The glass substrate of dimensions $15 \mathrm{~cm} \times 15 \mathrm{~cm}$ with a thickness of $5 \mathrm{~mm}$ was placed at the top of a cardboard box in such a way that the sunlight falls perpendicular to the plane of the glass plate. We carried out the experiment with both blank and mesoflower-coated glass substrates simultaneously. The temperature inside the cardboard boxes was measured using copper-constantan thermocouples. The measurements were done during a peak summer day at Chennai (longitude: $80^{\circ} 4^{\prime} 31^{\prime \prime} \mathrm{E}$, latitude: $13^{\circ}$ $00^{\prime} 19^{\prime \prime} \mathrm{N}$ ) at 1:00 p. m. (IST) where the intensity of sunlight was a maximum. The outside temperature was around $42{ }^{\circ} \mathrm{C}$ (the increase in temperature inside the enclosure compared to the outside during the measurements is largely due to the greenhouse effect). The data were collected every ten seconds and the experiments were carried out until the temperature inside the box became constant.

We did separate experiments with a monolayer and a bilayer of mesoflower-coated glass slides along with a blank glass substrate as the control. Compared with the blank glass substrate, the mesoflower monolayer-coated glass gave an average temperature inside the cardboard box which was lower by $2{ }^{\circ} \mathrm{C}$, whereas the bilayer-coated substrate showed a reduction of $4.3{ }^{\circ} \mathrm{C}$. Figures $7(\mathrm{~d})$ and $7(\mathrm{e})$ show the plots of variation of temperature inside the cardboard boxes as a function of exposure time for the monolayer and bilayer covered glass plate, respectively. The significant reduction in the temperature exhibited by the mesoflower-coated glass should be useful in the development of costeffective NIR-IR absorbing coatings for windows. We conducted a control experiment by using two blank glass plates of the same kind in the two cardboard boxes, which resulted in an almost identical temperatures inside both throughout the

\section{Springer}




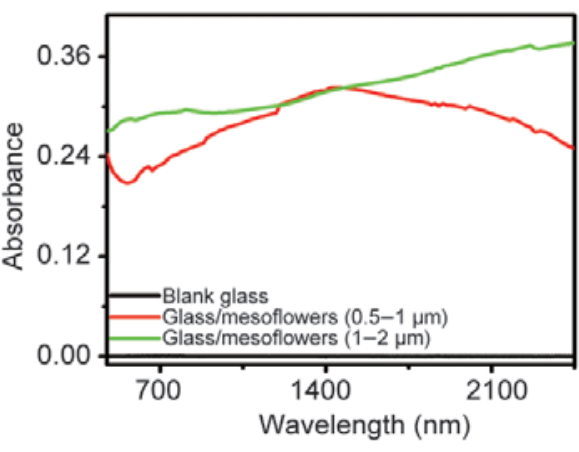

(a)

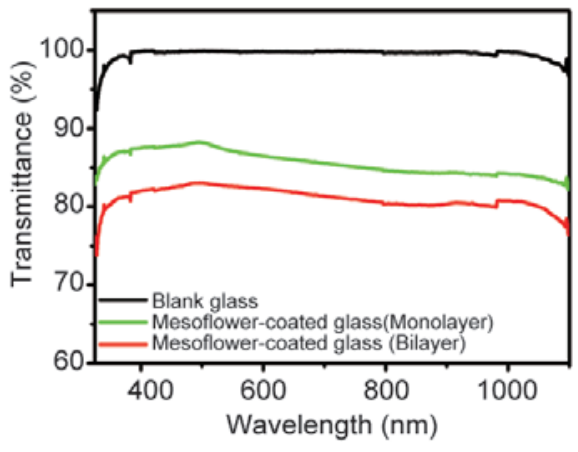

(b)
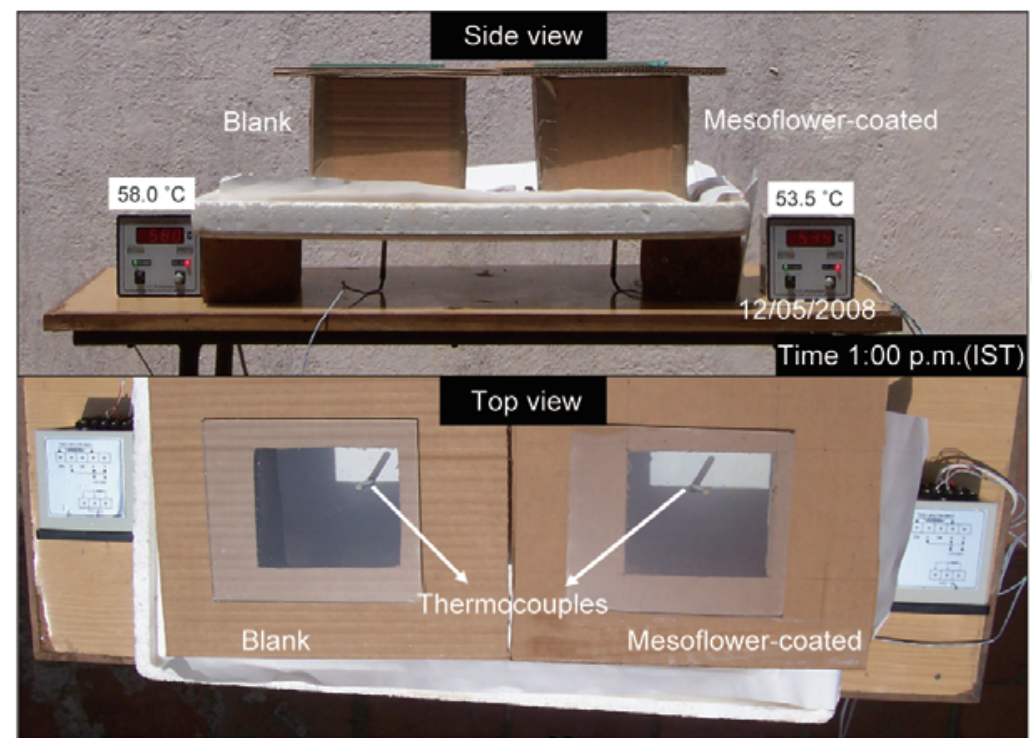

(c)

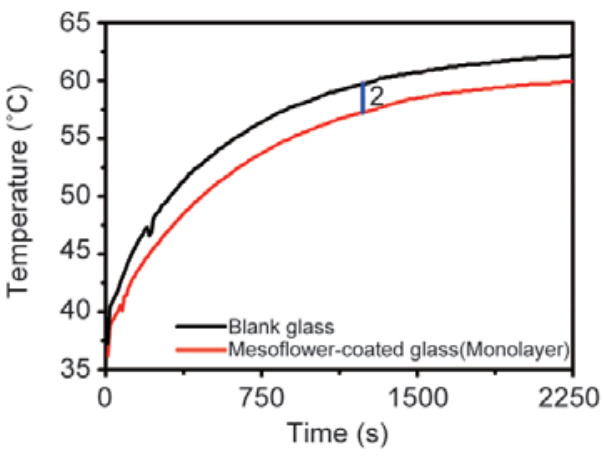

(d)

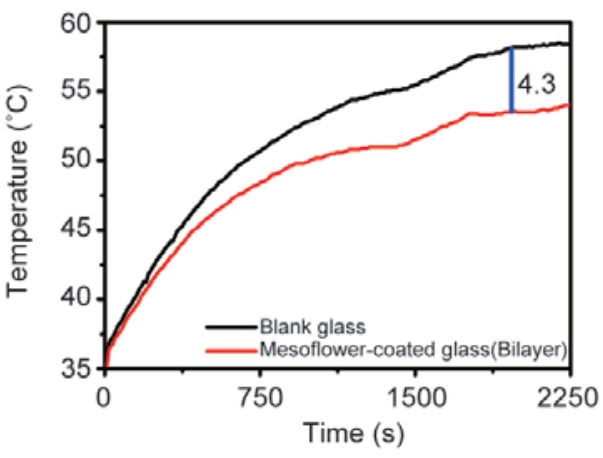

(e)

Figure 7 (a) UV-vis-NIR absorption spectra of monolayers of mesoflowers of various sizes and the corresponding spectrum of the blank glass substrate; (b) the transmittance spectra of the blank (black trace) as well as monolayer (green trace) and bilayer (red trace) of mesoflowers coated on the glass substrate; (c) photograph of the experimental set-up used for the real-time measurements of NIR-IR absorption properties of gold mesoflowers. The cardboard boxes are placed over a thermocol sheet. The thermocouple tip is at the centre of the box, as indicated in the picture. The thermocouple penetrates through the thermocol sheet. The whole set-up is kept on a table. Measurements were repeated with separate samples and on various days. (d) and (e) are plots of the variation of temperature inside the cardboard boxes as a function of exposure time for glass plates coated with a monolayer and bilayer of mesoflowers, respectively (the temperature fluctuations in (e) around $1500 \mathrm{~s}$ are due to the hindrance of the sunlight due to passing clouds). The initial increase in temperature, close to zero time, is very fast 
measurements (Fig. S-12 in the ESM).

It is known that the presence of spiky edges and tips enhances the electric field around nanoparticles. These tips are highly sensitive toward changes in the local dielectric environment. Theoretical predictions also show high SERS activity of such spiky nanoobjects [44]. We investigated the SERS activity of a monolayer of mesoflowers using crystal violet $(\mathrm{CV})$ as the analyte molecule and collected the Raman spectra of CV adsorbed on the mesoflowers at different concentrations (Fig. 8(a)). Interestingly we found that the material showed well-defined spectral features of $\mathrm{CV}$ even at a concentration of $10^{-10} \mathrm{~mol} / \mathrm{L}$. In order to compare the SERS activity of the mesoflowers with its spherical analogue, we collected the SERS spectra of CV molecules adsorbed on Au@citrate nanoparticles (NPs) [45]. Figure 8(b) shows the SERS spectra collected from CV adsorbed on NPs (traces 1 and 2) and from a blank glass plate

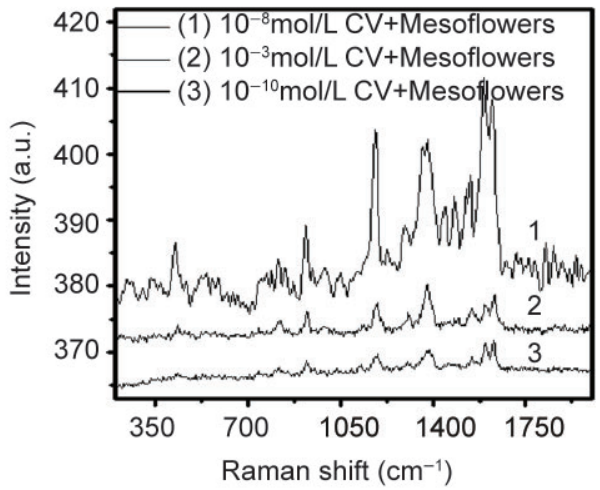

(a)

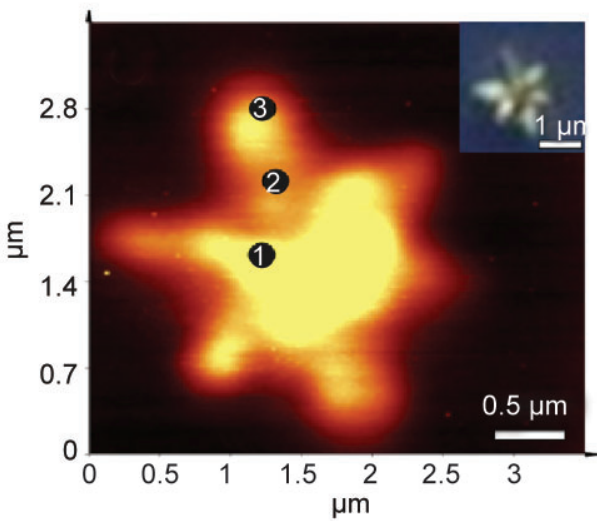

(c) (trace 3). The NP-coated substrate showed SERS signals down to a CV concentration of $10^{-6} \mathrm{~mol} / \mathrm{L}$. At a concentration of $10^{-7} \mathrm{~mol} / \mathrm{L}$, no distinct Raman signals were observed. In the case of $10^{-6} \mathrm{~mol} / \mathrm{L}$ of CV spotted on a blank glass plate, no Raman features were observed. The SERS enhancement factor was calculated for the mesoflowers as per a method in Ref. [46] and it was found to be $\sim 10^{9}$ for the 1593 $\mathrm{cm}^{-1}$ feature. The corresponding value for the NPs was of the order of $10^{4}$. The high SERS activity of the mesoflowers may be due to the large electric field enhancement at the sharp tips $[47,48]$ of each mesoflower as well as the "hot spots" [49] created in between the mesoflowers by the interlocking of two or more mesoflowers. The results indicate that these materials should be useful for making SERS-based sensors.

It was found that a single mesoflower particle can be observed using the Raman signals of adsorbed

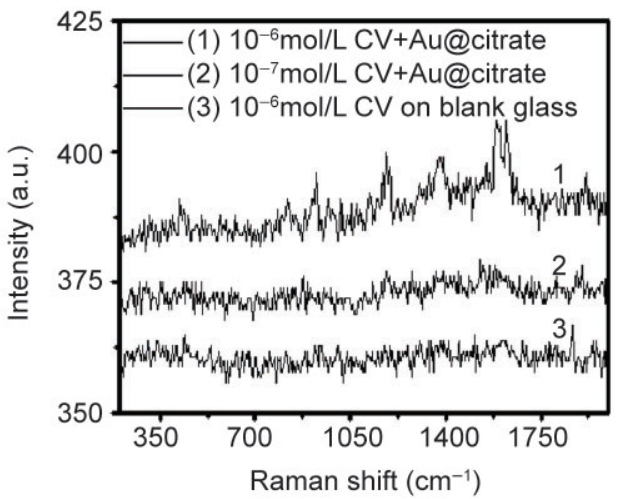

(b)

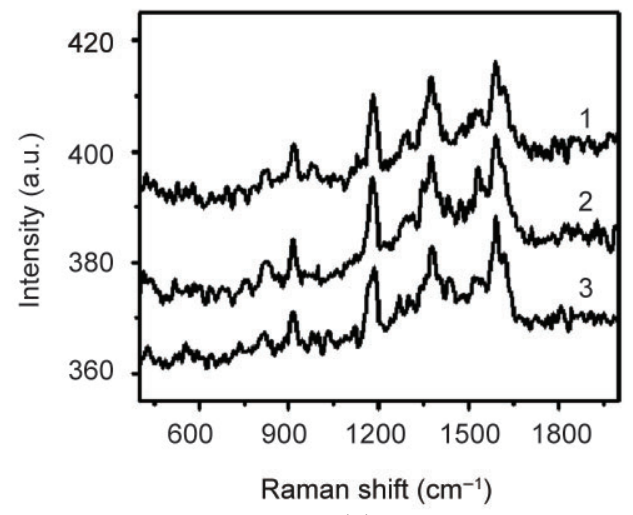

(d)

Figure 8 Raman spectra collected from CV solutions of different concentrations adsorbed on (a) mesoflower-coated glass substrate and (b) Au@citrate (spherical NPs)-coated glass substrate; (c) Raman image of a single mesoflower obtained by integrating Raman intensities of $10^{-6} \mathrm{~mol} / \mathrm{L} \mathrm{CV}$ solution in the 200-1800 $\mathrm{cm}^{-1}$ window; (d) single Raman spectra from various points marked in (c). The inset in (c) is an optical image of the mesoflower 
$\mathrm{CV}$ at a concentration of $10^{-6} \mathrm{~mol} / \mathrm{L}$. By collecting the SERS signals, ranging from 200 to $1800 \mathrm{~cm}^{-1}$, we imaged a single mesoflower (Fig. 8(c)). The corresponding optical image of the mesoflower is shown in the inset. The Raman spectra collected from the different areas of the mesoflower are shown in Fig. 8(d). It is clear from the intensities of the Raman features that the enhancement is almost the same for different areas over the mesoflower. This may be due to the unique morphology of the mesoflower and the electric field enhancement at the sharp edges and ridges on the mesoflowers. The Raman images of a single mesoflower taken at different depths proved the three-dimensional nature of the mesoflower (Fig. S-13 in the ESM). The unique morphology and the high SERS property of the mesoflowers can be utilized in security printing.

\section{Experimental}

\subsection{Materials}

Citric acid, tetrachloroauric acid trihydrate $\left(\mathrm{HAuCl}_{4}\right.$. $\left.3 \mathrm{H}_{2} \mathrm{O}\right)$, ascorbic acid, $\mathrm{AgNO}_{3}$, and $\mathrm{CTAB}$ were purchased from $\mathrm{CDH}$, India. Aniline, aminopropy trimethoxysilane (APTMS), and 1,9-nonanedithiol (NDT) were purchased from Sigma Aldrich. Triply distilled water was used throughout the experiments.

\subsection{Synthesis of gold mesoflowers}

The synthetic procedure used here is based on the seed-mediated growth method. All glassware was washed with aqua regia and rinsed with triply distilled water. Caution: Aqua regia is highly corrosive and must be handled carefully with proper protection. The seed nanoparticles used here for making mesoflowers were oligoaniline-capped Au nanoparticles which were synthesized as per our earlier procedure [25]. Briefly, $25 \mathrm{mg}$ of citric acid was dissolved in $35 \mathrm{~mL}$ of distilled water and maintained at $80{ }^{\circ} \mathrm{C} .1 \mathrm{~mL}$ of 25 $\mathrm{mmol} / \mathrm{L} \mathrm{HAuCl}_{4}$ was added to this solution. After the color changed from pale yellow to pink, $100 \mu \mathrm{L}$ distilled aniline was added immediately followed by $500 \mu \mathrm{L}$ of $25 \mathrm{mmol} / \mathrm{L} \mathrm{HAuCl}_{4}$. Heating was continued for 5 more min. This solution was kept at room temperature for $5 \mathrm{~h}$ and then centrifuged at $4000 \mathrm{rpm}$.
The resultant light pink supernatant solution, which contains Au / oligoaniline nanoparticles, was collected and used for further reaction.

In a typical synthesis of gold mesoflowers, 20 mL CTAB $(100 \mathrm{mmol} / \mathrm{L})$ was first heated to $80{ }^{\circ} \mathrm{C}$ in a round-bottomed flask over a heating mantle. To this solution, $335 \mu \mathrm{L} \mathrm{Au}^{3+}$ solution $(25 \mathrm{mmol} / \mathrm{L})$, $125 \mu \mathrm{L} \mathrm{AgNO}_{3}$ solution $(10 \mathrm{mmol} / \mathrm{L})$, and $135 \mu \mathrm{L}$ freshly prepared ascorbic acid solution $(100 \mathrm{mmol} / \mathrm{L})$ were added sequentially. $2 \mathrm{~mL}$ of Au/oligoaniline nanoparticles was added immediately to this growth solution and the solution gently mixed. No further stirring was carried out during the growth reaction. The resultant solution was kept undisturbed at $80{ }^{\circ} \mathrm{C}$ for $1 \mathrm{~h}$ and allowed to cool to room temperature in the laboratory atmosphere. After $1 \mathrm{~h}$, the solution was centrifuged at $4000 \mathrm{rpm}$ for $5 \mathrm{~min}$. The residue was washed with water three times, followed by methanol, in order to remove excess CTAB and other unwanted materials. The slight yellowish residue of gold mesoflowers was redispersed in deionized water and further characterized. This procedure yielded mesoflowers of size 1-2 $\mu \mathrm{m}$. Mesoflowers of $0.5^{-1} \mu \mathrm{m}$ and $\sim 0.15 \mu \mathrm{m}$ were synthesized by altering the reaction conditions: the above procedure was repeated by adding $5 \mathrm{~mL}$ and $6 \mathrm{~mL}$ of the precursor $\mathrm{Au} /$ oligoaniline nanoparticles, respectively, into 20 $\mathrm{mL}$ of the growth solution.

For the synthesis of meatball-like structures, $335 \mu \mathrm{L} \mathrm{Au}^{3+}$ solution $(25 \mathrm{mmol} / \mathrm{L}), 125 \mu \mathrm{L} \mathrm{AgNO}_{3}$ solution $(10 \mathrm{mmol} / \mathrm{L})$, and $135 \mu \mathrm{L}$ freshly prepared ascorbic acid solution ( $100 \mathrm{mmol} / \mathrm{L})$ were added sequentially to $20 \mathrm{~mL}$ of CTAB $(100 \mathrm{mmol} / \mathrm{L})$ solution in a beaker. To this growth solution, $2 \mathrm{~mL}$ of $\mathrm{Au}$ / oligoaniline nanoparticles was added. The beaker was immediately placed in an ice bath and kept there for $1 \mathrm{~h}$. After $1 \mathrm{~h}$, the solution was centrifuged at 4000 rpm for $5 \mathrm{~min}$. The residue was washed with water three times and characterized.

\subsection{Immobilization of the mesoflowers on a glass substrate}

The glass substrate was washed sequentially with a mild detergent solution, methanol and finally with pure water. It was then kept in a $10 \% \mathrm{HCl}$ solution for activation, washed with water, and dried under 
a stream of nitrogen. The glass plates were annealed at $350{ }^{\circ} \mathrm{C}$ for $4 \mathrm{~h}$ and cooled in a desiccator. They were then dipped in $50 \mathrm{mmol} / \mathrm{L}$ APTMS solution in methanol for $1 \mathrm{~h}$. Afterwards, they were washed with methanol and water in sequence and kept at $110{ }^{\circ} \mathrm{C}$ for $1 \mathrm{~h}$. The substrate was dipped in a gold mesoflower solution for $5 \mathrm{~h}$ and washed afterwards with distilled water and dried. The resultant mesoflower-coated substrate was further used for SEM, optical absorption, and SERS measurements. In order to make a bilayer of mesoflowers on a glass substrate, the monolayer-coated substrate was dipped in a $40 \mathrm{mmol} / \mathrm{L}$ solution of NDT in methanol for $3 \mathrm{~h}$. Subsequently this substrate was dipped in the mesoflower solution for $5 \mathrm{~h}$. This method of preparing a multilayer coating of nanoparticles has been used previously [50].

\subsection{Instrumentation}

Scanning electron microscope (SEM) images and EDAX studies were obtained using a Hitachi S-4800 FESEM and FEI Quanta-200 SEM. For the SEM measurements, samples were drop cast on an ITO conducting glass plate and dried (in addition to the covalent anchoring method described above). TEM was carried out using a JEOL 3011, $300 \mathrm{kV}$ instrument with a ultra-high resolution (UHR) pole piece. The samples for TEM were prepared by dropping the dispersion on amorphous carbon films supported on a copper grid and dried under ambient conditions. XPS measurements were conducted using an Omicron ESCA Probe spectrometer with monochromatized Al K $\alpha$ X-rays ( $h v=1486.6 \mathrm{eV})$. The samples were spotted as drop cast films on a sample stub, and the surfaces were scraped in situ to remove any surface contamination that could arise from components of the atmosphere, such as water and $\mathrm{CO}_{2} \cdot \mathrm{A}$ constant analyzer energy of $20 \mathrm{eV}$ was used for the measurements. LDI MS studies were done using a Voyager DE PRO Biospectrometry Workstation (Applied Biosystems) matrix assisted laser desorption ionization-time of flight mass spectrometer (MALDI-TOF MS). A pulsed nitrogen laser of $337 \mathrm{~nm}$ was used (maximum firing rate: 20 $\mathrm{Hz}$, maximum pulse energy: $300 \mu \mathrm{J}$ ) for the studies. Mass spectra were collected in positive and negative modes and were averaged for 100 shots. UV-vis-NIR measurements were carried out using a Varian 5E spectrometer in the range $200-2500 \mathrm{~nm}$. The optical absorbance measurements of films were carried out in the same way as in the case of solutions. Instead of using cuvettes, we used a mesoflower-coated glass plate and a glass plate of the same kind as the reference. Before taking the spectrum, baseline measurements were carried out using two blank glass plates of the same kind. The data are baseline corrected. The source beam was normal to the sample. X-ray diffraction (XRD) data were collected with a Shimadzu XD-D1 diffractometer using $\mathrm{Cu} \mathrm{K} \alpha$ radiation $(\lambda=1.54 \AA)$. The samples were scanned in the $2 \theta$ range $10^{\circ}-90^{\circ}$. Raman spectra were measured using a CRM 200 confocal Raman spectrometer (WiTec $\mathrm{GmbH}$ ). The substrate was mounted on the sample stage of the spectrometer. The SERS spectra were collected by exciting the sample with a $532 \mathrm{~nm}$ laser. The beam size used was $<1 \mu \mathrm{m}$. Data from liquid droplets were collected with a 60 $\times$ liquid immersion objective. The back-scattered light was collected by the $60 \times$ objective and sent to the spectrometer through a multimode fibre. The signals were then dispersed using a 600 grooves $/ \mathrm{mm}$ grating and the dispersed light was collected by a Peltier-cooled charge coupled device (CCD). For the Raman imaging, the mesoflower-coated glass substrate was dipped in $10^{-6} \mathrm{~mol} / \mathrm{L} \mathrm{CV}$ solution for $1 \mathrm{~h}$. The substrate was then washed with distilled water, dried, and mounted on the sample stage of the Raman spectrometer. The back-scattered light was collected by a $100 \times$ objective at an integration time of $50 \mathrm{~ms}$. Spectra from various part of the Raman image were collected and analyzed.

\section{Conclusions}

We haved prepared a new class of anisotropic mesostructured gold which can be called "mesoflowers". Although many other names such as nanothorns, nanourchins, nanostars, nanoflowers etc., can be thought of (the "nano" prefix could also be changed to "meso"), we prefer the term mesoflowers. Their complex morphology, uniformity, high degree of structural purity, and the ease of the synthetic

\section{算 Springer}


procedure and exciting properties make our material novel. The highly unsymmetrical mesoflower is made up of a large number of stems with unusual pentagonal symmetry. The synthetic methodology can yield uniform flowers of varying dimensions, ranging from nanometers to micrometers. It was found that temperature plays an important role in the formation of the mesoflowers. The utility of the mesoflowers as an infrared absorber is demonstrated by a simple real-time measurement. Preliminary results suggest that the mesoflowers can be used as infrared filters in architectural applications. SERS enhancement factors were determined for spherical NPs and mesoflowers. The high SERS activity of mesoflowers compared to their spherical analogues makes them ideal substrates for SERS as well as in other sensors. The unique morphology, high SERS activity, and possibility of Raman-based imaging of a single mesoflower make them good candidates for security applications.

\section{Acknowledgements}

We thank Department of Science and Technology, Government of India for constantly supporting our research program on nanomaterials. Prof. Keisaku Kimura, Department of Material Science, University of Hyogo, Japan is thanked for allowing the use of his FESEM. Dr. A. Sreekumaran Nair is thanked for assistance with the FESEM. T. S. Sreeprasad is thanked for help with the TEM measurements. Anshup is thanked for his assistance in IR absorption measurements. Robin John is thanked for help in the XPS measurements.

Electronic Supplementary Material: Supplementary material is available in the online version of this article at http://dx.doi.org/10.1007/s12274-009-9028-5 and is accessible free of charge.

\section{References}

[1] Xiao, Z. -L.; Han, C. Y.; Kwok, W. -K.; Wang, H. -H.; Welp, U.; Wang, J.; Crabtree. G. W. Tuning the architecture of mesostructures by electrodeposition. J. Am. Chem. Soc. 2004, 126, 2316-2317.
[2] Penner, R. M. Mesoscopic metal particles and wires by electrodeposition. J. Phys. Chem. B 2002, 106, 33393353.

[3] Sau, T. K.; Murphy, C. J. Seeded high yield synthesis of short Au nanorods in aqueous solution. Langmuir 2004, 20, 6414-6420.

[4] Sau, T. K.; Gole, A. M.; Orendorff, C. J.; Gao, J.; Gou, L.; Hunyadi, S. E.; Murphy, C. J. Anisotropic metal nanoparticles: Synthesis, assembly, and optical applications. J. Phys. Chem. B 2005, 109, 13857-13870.

[5] Perez-Juste, J.; Pastorìza-Santos, I.; Liz-Marzán, L. M.; Mulvaney, P. Gold nanorods: Synthesis, characterization and applications. Coord. Chem. Rev. 2005, 249, 18701901.

[6] Millstone, J. E.; Park, S.; Shuford, K. L.; Qin, L.; Schatz, G. C.; Mirkin, C. A. Observation of a quadrupole plasmon mode for a colloidal solution of gold nanoprisms. J. Am. Chem. Soc. 2005, 127, 5312-5313.

[7] Sajanlal, P. R.; Pradeep, T. Electric-field-assisted growth of highly uniform and oriented gold nanotriangles on conducting glass substrates. Adv. Mater. 2008, 20, 980983.

[8] Seong Ah, D. C.; Yun, Y. J.; Park, H. J.; Kim, W. J.; Ha, D. H.; Yun, W. S. Size-controlled synthesis of machinable single crystalline gold nanoplates. Chem. Mater. 2005, $17,5558-5561$.

[9] Jin, R.; Cao, Y.; Mirkin, C. A.; Kelly, K. L.; Schatz, G. C.; Zheng, J. G. Photoinduced conversion of silver nanospheres to nanoprisms. Science 2001, 294, 19011903.

[10] Hu, J.; Odom, T. W.; Lieber, C. M. Chemistry and physics in one dimension: Synthesis and properties of nanowires and nanotubes. Acc. Chem. Res. 1999, 32, 435-445.

[11] Wiley, B.; Sun, Y.; Mayers, B.; Xia. Y. Shape-controlled synthesis of metal nanostructures: The case of silver Chem. Eur. J. 2005, 11, 454-463.

[12] Hao, E.; Bailey, R. C.; Schatz, G. C.; Hupp, J. T.; Li, S. Synthesis and optical properties of "branched" gold nanocrystals. Nano Lett. 2004, 4, 327-330.

[13] Nehl, C. L.; Liao, H.; Hafner, J. H. Optical properties of star-shaped gold nanoparticles. Nano Lett. 2006, 6, 683 -688 .

[14] Hu, J.; Zhang, Y.; Liu, B.; Liu, J.; Zhou, H.; Xu, Y.; Jiang, Y. Y.; Yang, Z.; Tian, Z. Synthesis and properties of tadpoleshaped gold nanoparticles. J. Am. Chem. Soc. 2004, $126,9470-9471$. 
[15] Nishida, N.; Shibu, E. S.; Yao, H.; Oonishi, T.; Kimura, K.; Pradeep, T. Fluorescent gold nanoparticle superlattices. Adv. Mater. 2008, 20, 4719-4723.

[16] Yang, Y.; Liu, S.; Kimura, K. Superlattice formation from polydisperse Ag nanoparticles by a vapor-diffusion method. Angew. Chem. Int. Ed. 2006, 45, 5662-5665.

[17] Pastoriza-Santos, I.; Liz-Marzán, L. M. Synthesis of silver nanoprisms in DMF. Nano Lett. 2002, 2, 903-905.

[18] Liu, J.; Cankurtaran, B.; Wieczorek, L.; Ford, M. J.; Cortie, M. Anisotropic optical properties of semitransparent coatings of gold nanocaps. Adv. Funct. Mater. 2006, 16, 1457-1461.

[19] Maillard, M.; Giorgio, S.; Pileni, M. P. Silver nanodisks. Adv. Mater. 2002, 14, 1084-1086.

[20] El-Sayed, M. A. Some interesting properties of metals confined in time and nanometer space of different shapes. Acc. Chem. Res. 2001, 34, 257-264.

[21] Sreeprasad, T. S.; Samal, A. K.; Pradeep, T. Body- or tip-controlled reactivity of gold nanorods and their conversion to particles through other anisotropic structures. Langmuir 2007, 23, 9463-9471.

[22] Maier, S. A.; Brongersma, M. L.; Kik, P. G.; Meltzer, S.; Requicha, A. A. G.; Atwater, H. A. Plasmonics-A route to nanoscale optical devices. Adv. Mater. 2001, 13, 1501 -1505 .

[23] Shankar, S. S.; Rai, A.; Ankamwar, B.; Singh, A.; Ahmad, A.; Sastry, M. Biological synthesis of triangular gold nanoprisms. Nat. Mater. 2004, 3, 482-488.

[24] O'Neal, D. P.; Hirsch, L. R.; Halas, N. J.; Payne, J. D.; West, J. L. Photo-thermal tumor ablation in mice using near infrared-absorbing nanoparticles. Canc. Lett. 2004, 209, 171-176.

[25] Sajanlal, P. R.; Sreeprasad, T. S.; Nair, A. S.; Pradeep, T. Wires, plates, flowers, needles, and core-shells: Diverse nanostructures of gold using polyaniline templates. Langmuir 2008, 24, 4607-4614.

[26] Jena, B. K.; Raj, C. R. Seedless, surfactantless room temperature synthesis of single crystalline fluorescent gold nanoflowers with pronounced SERS and electrocatalytic activity. Chem. Mater. 2008, 20, 35463548.

[27] Bakshi, M. S.; Possmayer, F.; Petersen, N. O. Role of different phospholipids in the synthesis of pearl-necklacetype gold-silver bimetallic nanoparticles as bioconjugate materials. J. Phys. Chem. C 2007, 111, 14113-14124.

[28] Li, Y.; Shi, G. Electrochemical growth of two-dimensional gold nanostructures on a thin polypyrrole film modified ITO electrode. J. Phys. Chem. B 2005, 109, 2378723793.

[29] Jena, B. K.; Raj, C. R. Synthesis of flower-like gold nanoparticles and their electrocatalytic activity towards the oxidation of methanol and the reduction of oxygen. Langmuir 2007, 23, 4064-4670.

[30] Wang, W.; Cui, H. Chitosan-luminol reduced gold nanoflowers: From one-pot synthesis to morphologydependent SPR and chemiluminescence sensing. J. Phys. Chem. C 2008, 112, 10759-10766.

[31] Qian, L.; Yang, X. Polyamidoamine dendrimersassisted electrodeposition of gold-platinum bimetallic nanoflowers. J. Phys. Chem. B 2006, 110, 16672-16678.

[32] Yang, Z.; Lin, Z. H.; Tang, C. Y.; Chang, H. T. Preparation and characterization of flower-like gold nanomaterials and iron oxide/gold composite nanomaterials. Nanotechnology 2007, 18, 255606.

[33] Zijlstra, P.; Bullen, C.; Chon, J. W. M.; Gu, M. Hightemperature seedless synthesis of gold nanorods. J. Phys. Chem. B 2006, 110, 19315-19318.

[34] Fleming, D. A.; Williams, M. E. Size-controlled synthesis of gold nanoparticles via high-temperature reduction. Langmuir 2004, 20, 3021-3023.

[35] Huang, W. -L.; Chen, C. -H.; Huang, M. H. Investigation of the growth process of gold nanoplates formed by thermal aqueous solution approach and the synthesis of ultra-small gold nanoplates. J. Phys. Chem. C 2007, 111, 2533-2538.

[36] Park, H. J.; Ah, C. S.; Kim, W. -J.; Choi, I. S.; Lee, K. P. Temperature-induced control of aspect ratio of gold nanorods. J. Vac. Sci. Technol. A 2006, 24, 1323-1326.

[37] Elechiguerra, J. L.; Reyes-Gasga, J.; Yacaman, M. J. The role of twinning in shape evolution of anisotropic noble metal nanostructures. J. Mater. Chem. 2006, 16, 39063919.

[38] Chen, C.; Gao, Y. Electrosyntheses of poly(neutral red), a polyaniline derivative. Electrochim. Acta, 2007, 52, 3143 $-3148$.

[39] Wang, H. Y.; Mu, S. L. Bioelectrochemical characteristics of cholesterol oxidase immobilized in a polyaniline film. Sens. Actuators B 1999, 56, 22-30.

[40] Malinsky, M. D.; Kelly, K. L.; Schatz, G. C.; Van Duyne, R. P. Nanosphere lithography: Effect of substrate on the localized surface plasmon resonance spectrum of silver nanoparticles. J. Phys. Chem. B 2001, 105, 2343-2350.

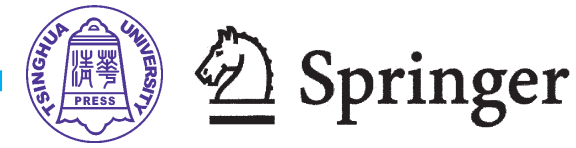


[41] Draine, B. T.; Flatau, P. J. Discrete-dipole approximation for scattering calculations. J. Opt. Soc. Am. A 1994, 11, 1491-1499.

[42] Jain, P. K.; Lee, K. S.; El-Sayed, I. H.; El-Sayed, M. A. Calculated absorption and scattering properties of gold nanoparticles of different size, shape, and composition: Applications in biological imaging and biomedicine. J. Phys. Chem. B 2006, 110, 7238-7248.

[43] Xu, X.; Stevens, M.; Cortie, M. B. In situ precipitation of gold nanoparticles onto glass for potential architectural applications. Chem. Mater. 2004, 16, 2259-2266.

[44] Kumar, P. S.; Pastoriza-Santos, I.; Rodríguez-González, B.; García de Abajo, F. J.; Liz-Marzán, L. M. Highyield synthesis and optical response of gold nanostars. Nanotechnology 2008, 19, 15606.

[45] Turkevich, J.; Stevenson, P. L.; Hillier, J. A study of the nucleation and growth processes in the synthesis of colloidal gold. Discuss. Faraday Soc. 1951, 11, 55-75.
[46] Kumar, G. V. P.; Shruthi, S.; Vibha, B.; Reddy, B. A. A.; Kundu, T. K.; Narayana, C. Hot spots in Ag core-Au shell nanoparticles potent for surface-enhanced Raman scattering studies of biomolecules. J. Phys. Chem. C 2007, 111, 4388-4392.

[47] Hao, E.; Schatz, G. C. Electromagnetic fields around silver nanoparticles and dimers. J. Chem. Phys. 2004, 120, 357-366.

[48] Hao, F.; Nehl, C. L.; Hafner, J. H.; Nordlander, P. Plasmon resonances of a gold nanostar. Nano Lett. 2007, 7, 729732.

[49] Qin, L.; Zou, S.; Xue, C.; Atkinson, A.; Schatz, G. C.; Mirkin, C. A. Designing, fabricating, and imaging Raman hot spots. Proc. Natl. Acad. Sci. USA 2006, 103, 1330013303.

[50] Subramaniam, C.; Chakrabarti, J.; Pradeep, T. Flowinduced transverse electrical potential across an assembly of gold nanoparticles. Phys. Rev. Lett. 2005, 95, 164501. 